\title{
Biomarkers in Her2- Positive Disease
}

\author{
Eva Valentina Klocker Christoph Suppan \\ Department of Oncology, Medical University of Graz, Graz, Austria
}

\section{Keywords}

Breast cancer $\cdot$ Her2 $\cdot$ Biomarker

\section{Abstract}

Background: Breast cancer is a heterogeneous disease with well-known characteristics such as hormone receptor (HR) status and human epidermal growth factor (Her)2 status. Although Her 2 represents an established treatment target, the development of resistance mechanisms during treatment, cardiotoxicity, and a worse response to standard therapies lead to worse outcomes. Summary: Therefore, we investigated various biomarkers in breast cancer such as Her2 mutations, Her2 heterogeneity, HR, PIK3CA, PTEN, programmed death receptor ligand 1 (PD-L1), tumor-infiltrating lymphocytes (TIL), micro RNA (miRNA), and BRCA mutations with regard to their clinical impact in Her2-positive disease. HR status and Her2 status, such as the presence of PIK3CA mutations, already play a role in treatment decision-making processes, whereas other biomarkers like PD-L1 status or TIL represent promising future markers. The influence of BRCA mutations in Her2-positive disease, Her2 mutations, and the impact of miRNA is vague to date. Antibody-drug conjugates (ADC) such as T-DM have been established as important treatment strategies, especially in Her2-positive disease. Key Message: However, up-to-date biomarkers appropriate for clinical practice are missing. Further studies are needed.

(c) 2020 S. Karger AG, Basel

\section{Introduction}

The transmembrane tyrosine kinase receptor Her2 (human epidermal growth factor receptor 2) has been intensively studied over the last decades and represents an established treatment target in immunotherapy of breast cancer in the neoadjuvant, adjuvant, and palliative settings.

Her2 is an epidermal growth factor receptor that is associated with a poor prognosis, such as earlier recurrence and metastatic disease in human breast cancer [1-3].

During the last few decades several treatment strategies targeting and blocking Her2 have been developed, such as monoclonal antibody trastuzumab as the first major achievement, and later the addition of pertuzumab, both blocking its extracellular domain, the kinase inhibitors lapatinib and neratinib, or the antibody-drug conjugate (ADC) trastuzumab emtansine [4-6].

The first established immunotherapy targeting Her2 was trastuzumab. In the adjuvant setting, trastuzumab significantly reduced the risk for recurrence or death [7]. Adding trastuzumab to chemotherapy in the metastatic setting, for example, prolongs the median survival from 20.3 to 25.1 months [8]. Nevertheless, acquired resistance to trastuzumab during therapy is a well-known and frequent phenomenon. Additionally, a serious adverse event of trastuzumab is its cardiotoxicity, especially in combination with anthracycline-based chemotherapy [9].

Finally, as studies have shown, blocking Her2 does not have the same effect in all Her2-positive breast cancers. As a result, biomarkers that distinguish between patients who would benefit from a treatment targeting Her2 and patients who would not derive such a benefit are demanded. 


\section{HER2 Receptor}

Her2 is a member of the epidermal growth factor receptor (EGFR) family which represents transmembrane growth factor receptors and includes the following 4 receptors: ErbB1, ErbB2, ErbB3, and ErbB4.

Consisting of 3 structures, i.e., the extracellular ligandbinding domain, the transmembrane domain, and the intracellular tyrosine kinase domain, these receptors are capable of transporting extracellular activation signals through the cell membrane [10]. Its activation affords the homodimerization of 2 intracellular kinase domains and transphosphorylation which leads to the activation of various intracellular second signal pathways $[10,11]$. Interestingly, one member of this family, i.e., the ErbB2 that encodes for Her2, is not activated by a direct ligand and builds heterodimeric complexes with other family members [12-14].

High membrane staining of Her2 (defined by the median of the immunohistochemistry [IHC]-modified $\mathrm{H}$ score as the cutoff) was associated with a significantly higher pCR rate in neoadjuvant treatment with trastuzumab, pertuzumab, and docetaxel compared to docetaxel and one immunotherapeutic substance or the combination of these 2 immunotherapeutic agents in the NeoSphere trial [15].

\section{Pathological Assessment}

Usually, IHC is used for Her 2 testing. Results such as IHC $0 / 1+$ can be treated like Her2-negative breast cancers, whereas IHC $3+$ results can be treated like Her2positive carcinomas. However, in IHC $2+$ tissues, the Her2 status is equivocal. In these cases with a membrane staining of $10-20 \%$, a reflex testing the HER2 gene expression by in situ hybridization (ISH) assay is advised by ASCO/CAP guidelines [16].

\section{Her2 Heterogeneity}

Her2 heterogeneity is defined by the presence of at least 2 distinct clones of cells with different levels of Her2 amplification within a tumor. The prevalence of heterogeneity is between 10 and 30\% [17-19].

A lower disease-free survival (DFS), a decreased overall survival (OS), and a worse response to trastuzumab in breast cancers with intratumoral Her2 heterogeneity were reported in a small cohort of 37 patients [20]. In mouse models, a significantly shorter survival and a higher incidence of metastases, compared to triple-negative (TN) and Her2-overexpressing breast cancers, have been reported in Her2-heterogeneous breast cancers [21].

The level of Her2 expression plays a prognostic role in metastatic breast cancer, as could be shown in the CLEOPATRA trial, where low Her2 levels (defined by the median of the IHC H-score as the cutoff) were associated with a worse progression-free survival (PFS) compared to high Her2 levels [22]. In addition, the level of Her2 expression was not predictive of the benefit of trastuzumab and pertuzumab compared to trastuzumab alone in combination with docetaxel [22]. On the other hand, T-DM1, which delivers the chemotherapy to tumor cells expressing the target, is supposed to be more dependent on Her2 levels [22]. However, there was no impact of Her2 levels on the relative effect of T-DM1 in the EMILIA trial or the TH3RESA trial, although the absolute median PFS was worse in patients with low Her2 levels assessed based on the median values of mRNA expression [23, 24].

Trastuzumab deruxtecan and trastuzumab duocarmazine are new anti-Her2 $\mathrm{ADC}$ and were shown to be active in low-Her2-expressing tumors (IHC $1+$ or $2+/$ not amplified) that are normally defined as Her2 negative [25, 26]. This superior effect compared to T-DM1 is caused by structural differences and a higher drug-to-antibody ratio.

Intratumoral heterogeneity was evaluated in several neoadjuvant trials and associated with non-pCR. Its potential to be negatively predictive for T-DM1 is still a matter of debate.

\section{Her2 Mutations}

Considering that acquired Her2 resistance is very common in Her2-positive breast cancers, we probably should pay more attention to mutations of Her2 which induce resistance.

The in-frame insertion of ErbB2, the P780-Y781 mutation, induces resistance to lapatinib by the AKT signaling pathway and leads to enhanced tumor growth [27].

Another ErbB2 mutation that leads to resistance to lapatinib is the gatekeeper mutation T798M [28]. Xu et al. [29] described Her2 reactivation through the L755S mutation during treatment with lapatinib and/or trastuzumab. Interestingly, in lobular breast cancers with Her2 amplification, CHD1 mutations were associated with significantly poorer DFS and OS [30].

Another interesting mutation of Her2 is the truncated p95Her2. The NH2 terminal fragment of the Her2 ectodomain p95Her2 is built through proteolytic shedding of Her2 (p815her2) and forms heterodimers with Her3 [31]. p95Her2 was reported as a significantly poor prognostic marker for DFS and OS in a cohort of 483 primary breast cancers, and it was significantly associated with a negative ER or PR status [32]. Interestingly, the full Her2 receptor (p815Her2) was not revealed as a significant prognostic marker either in the univariate or in the multivariate analysis in that study [32]. A previous study showed that p95Her2 was significantly associated with lymph node metastases, whereas p815Her2 was not [33].

p95Her2 was associated with a clinical response to lapatinib and capecitabine and a significantly longer PFS 
Fig. 1. PIK3CA status in the EMILIA and CLEOPATRA trials.

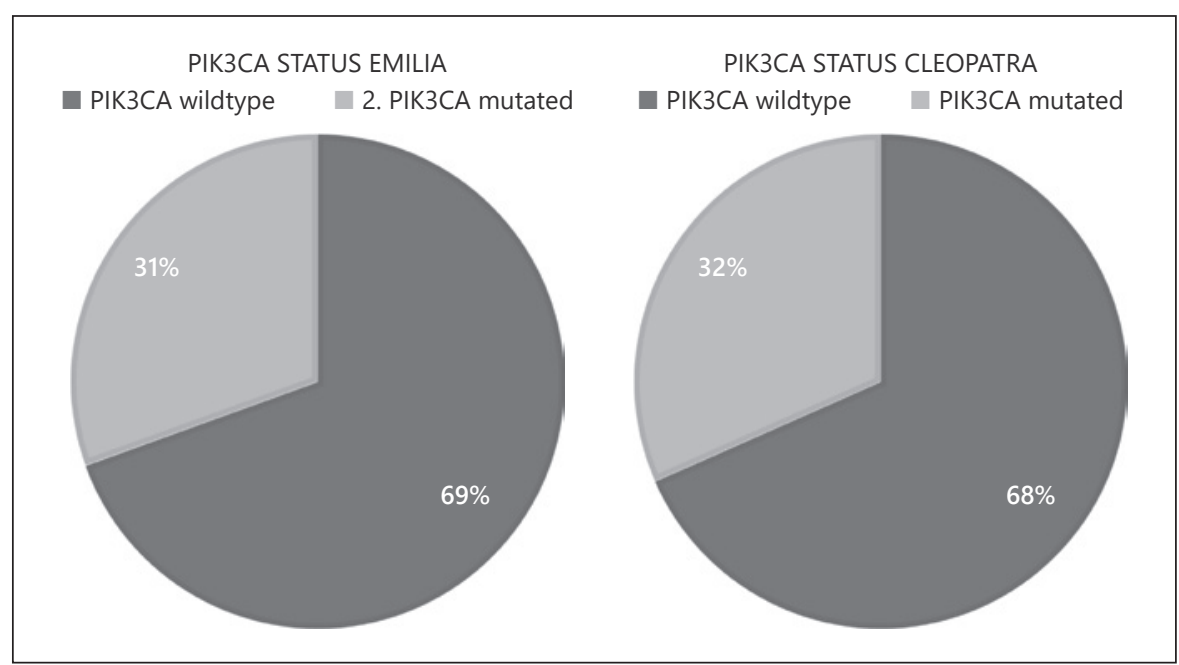

in ER-positive cases in trastuzumab-resistant breast cancers [34].

However, in order to establish mutation analyses of Her2 in routine clinical practice, further studies with larger populations are needed.

\section{Hormone Receptor}

To stratify different risk groups and plan the most suitable treatment, it is necessary to assess the hormone receptor (HR) and Her2 statuses. As a result, stratification in the following 4 distinct subgroups was established: luminal A, luminal B, Her2 overexpressing, and TN. Howlader et al. [35] showed, in their analyses of 196,094 invasive breast cancers between 2010 and 2013, 4-year survival rates of $92.5 \%$ in the $\mathrm{HR}+/ \mathrm{Her} 2-$ subgroup, $90.3 \%$ in the HR+/Her2+ subgroup, and $82.7 \%$ in the HR-/ Her2+ subgroup and the worst survival in the TN cohort with $77 \%$. Interestingly, in patients with stage 4 at time of diagnosis, the 4-year survival was better in the $\mathrm{HR}+1$ Her2+ cohort (45.5\%) than in the HR+/Her2- (35.9\%) subgroup [35]. The recurrence risk during the first 5 years was reported to be higher in the HR-/Her2+ subgroup compared to the $\mathrm{HR}+/ \mathrm{Her} 2+$ subgroup in stages $1-3$, but this effect disappeared after 5 years [36].

Parise et al. [3] investigated a large cohort of 143,333 stage 1-3 breast cancer patients between 2000 and 2010 and found that, within luminal B cancer, Her2-positivity was associated with a better 5 -year cumulative survival. The best survival within the luminal $\mathrm{B} / \mathrm{Her} 2$-positive subgroup was in the $\mathrm{ER}+/ \mathrm{PR}+/ \mathrm{Her} 2+$ group, while the poorest was in the ER-/PR+/Her2+ group [3]. Interestingly, in stage 3 , there was not a significant difference in cumulative survival between the $\mathrm{ER}+/ \mathrm{PR}+/ \mathrm{Her} 2+$ and the $\mathrm{ER}+/$ $\mathrm{PR}+/ \mathrm{Her} 2$ - groups [3]. Within the luminal $\mathrm{B} / \mathrm{Her} 2+$ subtypes, the ER-/PR+/Her2+ cohort was associated with the poorest OS in all stages [3]. In a study by Sáez et al. [32], including $483 \mathrm{Her} 2$-positive breast cancers, ER negativity was an independent poor prognostic marker for both PFS and OS, but PR negativity did not reach significance.

It has been reported that high ER levels and low Her2 levels are associated with a worse response to trastuzumab, for example in the HERA trial [37].

In the advanced setting, the use of CDK 4/6 inhibitors for $\mathrm{HR}+/ \mathrm{Her} 2+$ patients is of great interest. In the phase 2 trial monarcHER, the addition of abemaciclib to fulvestrant and trastuzumab showed a significant improvement of PFS in pretreated patients [38]. Data suggest that such combinations might overcome mechanisms of resistance to Her2-targeted therapies. However, biomarkers beyond ER and Her2 status have to be identified to select those patients who might have the greatest benefit of the combination of CDK 4/6 inhibition, endocrine therapy, and Her2-targeted therapy.

\section{PI3K/Akt/mTOR Pathway}

Heterodimerization of Her2 activates the RAS pathway, e.g., the PI3K/Akt/mTOR pathway, which plays an important role in cell proliferation, apoptosis, or growth [39]. Activating mutations in the PIK3CA gene lead to a higher activity of this pathway, especially of Akt, and have been detected in breast cancer cells $[39,40]$. Another reason for dysregulation of this pathway is the inactivating mutation of the tumor suppressor gene PTEN [41].

There is evidence that the Her3 family member plays a special role in this pathway. It builds the heterodimer with Her2 that activates the PI3K/Akt/mTOR cascade [42].

\section{PIK3CA}

Saal et al. found a significant correlation between PIK3CA mutations and expression of ER, PR, and Her2 and lymph node metastases and the PTEN wild type [43].

In a biomarker analysis of the CELOPATRA trial, PIK3CA mutations pooled as exons 20 and 9 were re- 
vealed as poor prognostic markers and significantly associated with a shorter PFS in both the trastuzumab/pertuzumab/doxorubicin group and the control group with trastuzumab/placebo/doxorubicin in the metastatic setting [22]. In the metastatic setting in the EMILIA trial, PIK3CA mutations (exons 1, 4, 7, 9, and 20) were associated with a shorter OS and PFS only in the capecitabineplus-lapatinib group, but not in the T-DM1 group [23]. In multivariate analyses, PIK3CA mutations were revealed as an independent poor prognostic marker for OS in the T-DM1 subgroup [23]. In both trials PIKCA mutations were detected in about $30 \%$ of the cases (Fig. 1).

These results were supported by a small study including 80 patients in the metastatic setting after treatment with trastuzumab under actual treatment with lapatinib plus capecitabine [34]. Additionally, patients with PIK3CA mutations (exons 9 and 20) showed a significantly shorter PFS in the ER-positive subgroup but not in the ER-negative group [34].

In the NeoSphere trial evaluating neoadjuvant combinations of pertuzumab, trastuzumab, and docetaxel in 417 patients, only exon 9 PIK3CA mutations (within exons 20, 7, and 9) were associated with worse pCR rates [15]. However, the population was too small to evaluate the impact of exon 7 mutations [15]. Interestingly, ER positivity did not show an effect on PCR in PIK3CA-mutated tumors in the NeoSphere trial [15].

In another biomarker analysis including 504 Her2positive breast cancer patients from 3 studies, i.e., GeparQuattro, GeparQuinto, and GeparSixto, PIK3CA mutations (exons 9 and 20) were significantly associated with worse pCR rates in HR-positive breast cancers but not in HR-negative ones in all of the trials and in the GeparSixto study subgroup [44]. In a multivariate analysis, PIK3CA mutations and HR positivity were independently adversely associated with pCR in all patients and in the GeparSixto trial [44]. After stratification for treatment with lapatinib, trastuzumab, and the combination of trastuzumab and lapatinib, especially the PIK3CA wildtype subgroups receiving trastuzumab and its combination with lapatinib showed significantly higher $\mathrm{pCR}$ rates than PIK3CA-mutated tumors ( $p=0.017)$ [44]. However, for OS only a trend toward a poor impact of PIK3CA mutations was detected [44]. A small study with 121 neoadjuvant patients by Guarneri et al. [45] showed no significant influence of PIK3CA status regarding pCR rates in patients receiving trastuzumab and only a tendency in treatment with trastuzumab and lapatinib.

In the adjuvant FinHER adjuvant trial including 1,010 breast cancer patients, PIK3CA mutations (exons 1, 2, 4, 9, 13, 18, and 20) were associated with ER positivity, luminal A subtype, histological grade, and tumor stage [46]. Controversially, there was no difference in the treatment of PIK3CA-mutant patients and wild-type patients treat- ed with trastuzumab, and no effect regarding DFS could be observed [46].

\section{PTEN}

Although PTEN loss plays an important role in tumor growth, its clinical significance as a predictive marker is controversial. In the EMILIA trial PTEN was not revealed as a prognostic marker [23]. Nevertheless, an enhanced effect on PFS in the PTEN-decreased subgroup receiving T-DM1 versus the lapatinib/capecitabine subgroup compared to the PTEN wild-type cohort was detected [23]. This effect could not be shown for OS, though [23].

Interestingly, Tekesin et al. [47] found a significant association of PTEN loss with poor response rates with trastuzumab and a shorter PFS in 100 Her2-positive metastatic breast cancer patients, whereas PIK expression did not show any effect. In the metastatic setting in a small cohort of 45 patients, no significant association between response to trastuzumab and PTEN status was found [48]. However, PTEN status had been differently defined in those studies.

In the adjuvant North Central Cancer Treatment Group N9831 Trial, PTEN positivity, defined as cytoplasmatic staining, IHC $1+$ to $3+$ was associated with a positive lymph node status and ER negativity, but no association with DFS or OS could be reported [49]. In a neoadjuvant trial by Nuciforo et al. [50], no impact of PTEN status on pCR rates after neoadjuvant treatment with lapatinib or trastuzumab or the combination could be defined.

\section{$P D-L 1$}

In a study to investigate the impact of programmed death ligand 1 (PD-L1) in breast cancer by Bae et al. [51], PD-L1 positivity (defined using a modified Muenst scoring method) was significantly correlated to histological grade, a negative lymph node stage, pathological stage, Her2 positivity, TN subtype, ER and PR negativity, and an abundance of tumor-infiltrating lymphocytes (TIL) [51].

In Her2-positive breast cancer receiving adjuvant chemotherapy and Her2-blocking therapy, high PD-L1 (defined using a modified Muenst scoring method) and TIL were associated with a better OS and DFS in Kaplan-Meier analysis and univariate analysis [51]. In multivariate analysis, only the abundance of TIL remained as an independent prognostic marker [51]. Interestingly, in a study by Tsang et al. [52] including 1,091 patients at all stages, high PD-L1 (defined by the mean score as the cutoff) in Her2-positive, HR-negative disease was revealed as an independent poor prognostic marker, especially in combination with low PD-TIL [52].

In a cohort of 126 Her2-positive breast cancer patients receiving neoadjuvant treatment with trastuzumab, $\mathrm{PD}$ - 
L1 positivity ( $\geq 1 \%$ ) was associated with high TIL, high levels of CD8-positive lymphocytes, and histological grade 3 [53]. In univariate analysis, high PD-L1 levels were associated with high pCR rates. However, this result could not be confirmed in the multivariate analysis [53]. Additionally, PD-L1 positivity did not show an effect on recurrence-free survival, but in PD-L1-negative, Her2positive cases, high CD8-positive lymphocytes were associated with a longer recurrence-free survival [53].

In the PANACEA trial, a response due to the addition of pembrolizumab to trastuzumab in advanced, unresectable, or metastatic breast cancers after progression under trastuzumab was shown in PD-L1 positivity $(\geq 1 \%)$, which additionally correlated to TIL [54]. Hou et al. [55] showed a significant longer OS in PD-L1-expressing $(\geq 1 \%)$ and $\mathrm{CD}^{+}$-infiltrating tumor lymphocytes in Kaplan-Meier and univariate analyses.

\section{Tumor-Infiltrating Lymphocytes}

Kochi et al. [56] showed an association of a high expression of TIL genomic signature with a better outcome regarding distant event-free survival in a small cohort of Her2-positive breast cancer patients $(n=120)$ [56]. Interestingly, this effect could not be shown in luminal or TN breast cancer [56]. On the other hand, higher pCR rates after neoadjuvant chemotherapy in patients with a high TIL genomic signature have been detected in luminal breast cancer, Her2-positive disease, and TN breast cancer [56].

Additionally, there is more evidence for a relation between high TIL and an increased benefit of chemotherapy or immunotherapy with trastuzumab $[57,58]$. The association of higher TIL with a higher pCR could be estimated in the neoadjuvant setting $[59,60]$. Furthermore, in Her2-positive patients with low stromal TIL $<10 \%$ administration of trastuzumab did not significantly impact pCR rates or PFS [61]. However, it remains unclear whether the impact of high TIL on PCR rates relates to the treatment with trastuzumab [59]. A study by Liu et al. [62] revealed the presence of intratumoral CD8 ${ }^{+}$TIL detected in a tumor microarray as an independent prognostic factor for breast cancer survival in a cohort of 3,144 breast cancer patients of all types. The prevalence for intratumoral TIL was associated with a younger age, ER negativity, Her2 positivity, a high grade, medullary histology, and the core basal breast cancer subgroup [62]. However, in Her2+/ER- there was only a trend toward improvement but not a significant association with breast cancer-specific survival [62].

However, the most appropriate method for the assessment of TIL remains unclear. Some authors used tissue microarrays, whereas others used histopathological material from biopsies or tissues from surgical resection. Additionally, it is not clear whether intratumoral or stromal
Table 1. Prediction of the biomarkers

\begin{tabular}{ll}
\hline Biomarker & Prediction \\
\hline $\begin{array}{l}\text { Her2 heterogeneity } \\
\text { Her2 expression }\end{array}$ & Worse DFS and OS [20, 21] \\
Her2 mutations & Better PFS [22-24] \\
P780-Y781 & Resistance to lapatinib [27] \\
T798M & Resistance to lapatinib [28] \\
L755S & Her2 reactivation during \\
& treatment [29] \\
CHD 1 & ILC worse OS and DFS [30] \\
P95Her2 & Worse OS and DFS [32] \\
Hormone receptor & Better OS and DFS [35, 36, 38] \\
ER positivity & Better OS and DFS [3, 37] \\
PR positivity & Unclear [3, 32] \\
PIK3CA mutation & Controversial data [15, 22, 23, \\
& 34, 43-46] \\
PTEN & No effect [23, 47-50] \\
PD-L1 & Controversial data [51-54] \\
TIL & Higher pCR rates \\
& Association with better DFS \\
BRCA & and OS [51, 56, 59, 60] \\
miRNA & No data about its prognostic \\
Downregulation of miR-342 & impact up to date \\
Upregulation of miR-221 & Resistance to tamoxifen [67] \\
Upregulation of miR-222 & Resistance to tamoxifen [68] \\
\hline
\end{tabular}

TIL should be assessed. However, it has not been determined whether these different assessments could be compared. Altogether, the presence of TIL seems to be an indicator of the response to chemotherapy. However, its independent prognostic impact is unclear $[59,60]$.

Taking this data into account, Her2-positive disease seems to be next after TN breast cancer, where immunomodulation and immune checkpoint inhibitors may be developed further in the clinics [63].

\section{$B R C A$}

BRCA mutations are rare among Her2-positive breast cancers $[64,65]$. In a study by Evans et al. [65], in Her2positive breast cancers, only $5.8 \%$ (9 out of 156) of the patients carried a BRCA mutation and just $6.8 \%$ of patients with hereditary BRCA2 mutations were Her2-positive, with $2.1 \%$ among the BRCA 1-mutated subgroup [65]. However, to our knowledge, its impact on prognosis had not been assessed yet.

\section{Micro RNA}

Micro RNA (miRNA) are short, single-stranded, noncoding RNA that play a role by targeting messenger RNA. As they can be detected in breast cancer tissues of early breast cancers such as in the blood of metastatic tumor patients, they might represent useful biomarkers [66]. 
Lowery et al. [67] showed the possibility to predict HR positivity or Her2 positivity by miRNA. Her2 positivity in breast cancer could be identifiable with the signature of miR-520d, miR-181c, miR-302c, miR-376b, and miR30e [67]. Additionally, miR-342, which was revealed as a marker for ER positivity, was associated with Her2 positivity [67]. Interestingly, in another study comparing the tamoxifen-sensitive cell line MCF-7 with the tamoxifenresistant cell line OHTR, the downregulated miR-342 was a marker for tamoxifen resistance [68]. Upregulation of miR-221 and miR-222 in human breast cancer cells was associated with Her2-positivity and could induce tamoxifen resistance in MCF-7 cells [68]. Further studies are needed to evaluate the clinical relevance of miRNA.
Biomarkers to find the most suitable treatment strategy for each patient are needed. However, further studies will be necessary to find a biomarker appropriate for daily routine.

\section{Acknowledgement}

We acknowledge Klara Balic for her grammar review.

\section{Conflict of Interest Statement}

E.V.K. received a travel grant from Pierre Fabre. C.S. received invitations for lectures from Pfizer, Roche, and Novartis and travel grants from Pierre Fabre, Novartis, Pfizer and Roche.

\section{Funding Sources}

There are no applicable funding sources.

\section{Author Contributions}

E.V.K. and C.S. wrote this paper. well as the tumor-immune microenvironment of Her2positive breast cancer, with its potential for the efficacy of immune checkpoint inhibitors, are promising.

\section{References}

1 Paterson MC, Dietrich KD, Danyluk J, Paterson AH, Lees AW, Jamil N, et al. Correlation between c-erbB-2 amplification and risk of recurrent disease in node-negative breast cancer. Cancer Res. 1991 Jan;51(2):556-67.

2 Ross JS, Fletcher JA. The HER-2/neu oncogene in breast cancer: prognostic factor, predictive factor, and target for therapy. Stem Cells. 1998;16(6):413-28.

3 Parise CA, Caggiano V. Breast cancer survival defined by the ER/PR/HER2 subtypes and a surrogate classification according to tumor grade and immunohistochemical biomarkers. J Cancer Epidemiol. 2014;2014: 469251.

4 Hudis CA. Trastuzumab: mechanism of action and use in clinical practice. N Engl J Med. 2007 Jul;357(1):39-51.

5 Martin M, Holmes FA, Ejlertsen B, Delaloge S, Moy B, Iwata H, et al.; ExteNET Study Group. Neratinib after trastuzumab-based adjuvant therapy in HER2-positive breast cancer (ExteNET): 5-year analysis of a randomised, double-blind, placebo-controlled, phase 3 trial. Lancet Oncol. 2017 Dec;18(12): 1688-700.

6 Diéras V, Miles D, Verma S, Pegram M, Welslau M, Baselga J, et al. Trastuzumab emtansine versus capecitabine plus lapatinib in patients with previously treated HER2-positive advanced breast cancer (EMILIA): a de- scriptive analysis of final overall survival results from a randomised, open-label, phase 3 trial. Lancet Oncol. 2017 Jun;18(6):732-42.

7 Cameron D, Piccart-Gebhart MJ, Gelber RD, Procter M, Goldhirsch A, de Azambuja E, et al.; Herceptin Adjuvant (HERA) Trial Study Team. 11 years' follow-up of trastuzumab after adjuvant chemotherapy in HER2-positive early breast cancer: final analysis of the HERceptin Adjuvant (HERA) trial. Lancet. 2017 Mar;389(10075):1195-205.

8 Slamon DJ, Leyland-Jones B, Shak S, Fuchs H, Paton V, Bajamonde A, et al. Use of chemotherapy plus a monoclonal antibody against HER2 for metastatic breast cancer that overexpresses HER2. N Engl J Med. 2001 Mar; 344(11):783-92.

9 Moja L, Tagliabue L, Balduzzi S, Parmelli E, Pistotti V, Guarneri V, et al. Trastuzumab containing regimens for early breast cancer. Cochrane Database Syst Rev. 2012 Apr 18; 2012(4):CD006243.

10 Moasser MM. The oncogene HER2: its signaling and transforming functions and its role in human cancer pathogenesis. Oncogene. 2007 Oct;26(45):6469-87.

$11 \mathrm{CaH}$. Trastuzumab: mechanism of action and use in clinical practice.

12 Yarden Y, Sliwkowski MX. Untangling the ErbB signalling network. Nat Rev Mol Cell Biol. 2001 Feb;2(2):127-37.
13 Cho HS, Mason K, Ramyar KX, Stanley AM, Gabelli SB, Denney DW Jr, et al. Structure of the extracellular region of HER2 alone and in complex with the Herceptin Fab. Nature. 2003 Feb;421(6924):756-60.

14 Garrett TP, McKern NM, Lou M, Elleman TC, Adams TE, Lovrecz GO, et al. The crystal structure of a truncated ErbB2 ectodomain reveals an active conformation, poised to interact with other ErbB receptors. Mol Cell. 2003 Feb;11(2):495-505.

15 Bianchini G, Kiermaier A, Bianchi GV, Im YH, Pienkowski T, Liu MC, et al. Biomarker analysis of the NeoSphere study: pertuzumab, trastuzumab, and docetaxel versus trastuzumab plus docetaxel, pertuzumab plus trastuzumab, or pertuzumab plus docetaxel for the neoadjuvant treatment of HER2-positive breast cancer. Breast Cancer Res. 2017 Feb;19(1):16.

16 Wolff AC, Hammond ME, Allison KH, Harvey BE, Mangu PB, Bartlett JM, et al. Human Epidermal Growth Factor Receptor 2 Testing in Breast Cancer: American Society of Clinical Oncology/College of American Pathologists Clinical Practice Guideline Focused Update. J Clin Oncol. 2018 Jul;36(20):2105-22.

17 Vance GH, Barry TS, Bloom KJ, Fitzgibbons PL, Hicks DG, Jenkins RB, et al. Genetic heterogeneity in HER2 testing in breast cancer: panel summary and guidelines. 
18 Hanna WM, Rüschoff J, Bilous M, Coudry RA, Dowsett M, Osamura RY, et al. HER2 in situ hybridization in breast cancer: clinical implications of polysomy 17 and genetic heterogeneity. Mod Pathol. 2014 Jan;27(1):4-18.

19 Lee HJ, Seo AN, Kim EJ, Jang MH, Suh KJ, Ryu HS, et al. HER2 heterogeneity affects trastuzumab responses and survival in patients with HER2-positive metastatic breast cancer. Am J Clin Pathol. 2014 Dec;142(6): 755-66.

20 Rye IH, Trinh A, Saetersdal AB, Nebdal D, Lingjaerde OC, Almendro V, et al. Intratumor heterogeneity defines treatment-resistant HER2+ breast tumors. Mol Oncol. 2018 Nov;12(11):1838-55.

21 Hosonaga M, Arima Y, Sampetrean O, Komura D, Koya I, Sasaki T, et al. HER2 Heterogeneity Is Associated with Poor Survival in HER2-Positive Breast Cancer. Int J Mol Sci. 2018 Jul;19(8):2158.

22 Baselga J, Cortés J, Im SA, Clark E, Ross G, Kiermaier A, et al. Biomarker analyses in CLEOPATRA: a phase III, placebo-controlled study of pertuzumab in human epidermal growth factor receptor 2-positive, firstline metastatic breast cancer. J Clin Oncol. 2014 Nov;32(33):3753-61.

23 Baselga J, Lewis Phillips GD, Verma S, Ro J, Huober J, Guardino AE, et al. Relationship between tumor biomarkers and efficacy in EMILIA, a phase III study of trastuzumab emtansine in HER2-positive metastatic breast cancer. Clin Cancer Res. 2016 Aug;22(15):3755-63.

24 Kim SB, Wildiers H, Krop IE, Smitt M, Yu R, Lysbet de Haas S, et al. Relationship between tumor biomarkers and efficacy in TH3RESA, a phase III study of trastuzumab emtansine (T-DM1) vs. treatment of physician's choice in previously treated HER2-positive advanced breast cancer. Int J Cancer. 2016 Nov; 139(10):2336-42.

25 Modi S, Saura C, Yamashita T, Park YH, Kim SB, Tamura K, et al.; DESTINY-Breast01 Investigators. Trastuzumab Deruxtecan in previously treated HER2-positive breast cancer. N Engl J Med. 2020 Feb;382(7):610-21.

26 Banerji U, van Herpen CM, Saura C, Thistlethwaite F, Lord S, Moreno V, et al. Trastuzumab duocarmazine in locally advanced and metastatic solid tumours and HER2-expressing breast cancer: a phase 1 dose-escalation and dose-expansion study. Lancet Oncol. 2019 Aug;20(8):1124-35.

27 Yang ZY, Yang L, Xu CW, Wang XJ, Lei L. An insertion mutation of ERBB2 enhances breast cancer cell growth and confers resistance to lapatinib through AKT signaling pathway. Biol Open. 2020 Jan;9(1):bio047662. https:// doi.org/10.1242/bio.047662.

28 Meng X, Li Y, Tang H, Mao W, Yang H, Wang $\mathrm{X}$, et al. Drug response to HER2 gatekeeper T798M mutation in HER2-positive breast cancer. Amino Acids. 2016 Feb;48(2):487-97.

$29 \mathrm{Xu}$ X, De Angelis C, Burke KA, Nardone A, $\mathrm{Hu}$ H, Qin L, et al. HER2 Reactivation through Acquisition of the HER2 L755S Mutation as a Mechanism of Acquired Resistance to HER2targeted Therapy in HER2+ Breast Cancer. Clin Cancer Res. 2017 Sep;23(17):5123-34.
30 Ping Z, Siegal GP, Harada S, Eltoum IE, Youssef M, Shen T, et al. ERBB2 mutation is associated with a worse prognosis in patients with $\mathrm{CDH} 1$ altered invasive lobular cancer of the breast. Oncotarget. 2016 Dec;7(49): 80655-63.

31 Xia W, Liu LH, Ho P, Spector NL. Truncated ErbB2 receptor (p95ErbB2) is regulated by heregulin through heterodimer formation with ErbB3 yet remains sensitive to the dual EGFR/ErbB2 kinase inhibitor GW572016. Oncogene. 2004 Jan;23(3):646-53.

32 Sáez R, Molina MA, Ramsey EE, Rojo F, Keenan EJ, Albanell J, et al. p95HER-2 predicts worse outcome in patients with HER2 -positive breast cancer. Clin Cancer Res. 2006 Jan;12(2):424-31.

33 Molina MA, Saez R, Ramsey EE, GarciaBarchino M-J, Rojo F, Evans AJ, et al. NH2terminal truncated HER-2 protein but not full-length receptor is associated with nodal metastasis in human breast cancer. Clin Cancer Res. 2002 Feb;8(2):347-53.

34 Nishimura R, Toh U, Tanaka M, Saimura M, Okumura Y, Saito T, et al. Role of HER2-Related Biomarkers (HER2, p95HER2, HER3, PTEN, and PIK3CA) in the Efficacy of Lapatinib plus Capecitabine in HER2-Positive Advanced Breast Cancer Refractory to Trastuzumab. Oncology. 2017;93(1):51-61.

35 Howlader N, Cronin KA, Kurian AW, Andridge R. Differences in Breast Cancer Survival by Molecular Subtypes in the United States. Cancer Epidemiol Biomarkers Prev. 2018 Jun;27(6):619-26.

36 Vaz-Luis I, Ottesen RA, Hughes ME, Marcom PK, Moy B, Rugo HS, et al. Impact of hormone receptor status on patterns of recurrence and clinical outcomes among patients with human epidermal growth factor-2-positive breast cancer in the National Comprehensive Cancer Network: a prospective cohort study. Breast Cancer Res. 2012 Oct; 14(5):R129.

37 Loi S, Dafni U, Karlis D, Polydoropoulou V, Young BM, Willis S, et al. Effects of Estrogen Receptor and Human Epidermal Growth Factor Receptor-2 Levels on the Efficacy of Trastuzumab: A Secondary Analysis of the HERA Trial. JAMA Oncol. 2016 Aug;2(8): 1040-7.

38 Tolaney SM, Wardley AM, Zambelli S, Hilton JF, Troso-Sandoval TA, Ricci F, et al. Abemaciclib plus trastuzumab with or without fulvestrant versus trastuzumab plus standardof-care chemotherapy in women with hormone receptor-positive, HER2-positive advanced breast cancer (monarcHER): a randomised, open-label, phase 2 trial. Lancet Oncol. 2020 Jun;21(6):763-75.

39 Vivanco I, Sawyers CL. The phosphatidylinositol 3-Kinase AKT pathway in human cancer. Nat Rev Cancer. 2002 Jul;2(7):489-501.

40 Samuels Y, Wang Z, Bardelli A, Silliman N, Ptak J, Szabo S, et al. High frequency of mutations of the PIK3CA gene in human cancers. Science. 2004 Apr;304(5670):554-554.

41 Li J, Yen C, Liaw D, Podsypanina K, Bose S, Wang SI, et al. PTEN, a putative protein tyrosine phosphatase gene mutated in human brain, breast, and prostate cancer. Science. 1997 Mar;275(5308):1943-7.
42 Holbro T, Beerli RR, Maurer F, Koziczak M, Barbas CF 3rd, Hynes NE. The ErbB2/ErbB3 heterodimer functions as an oncogenic unit: ErbB2 requires ErbB3 to drive breast tumor cell proliferation. Proc Natl Acad Sci USA. 2003 Jul;100(15):8933-8.

43 Saal LH, Holm K, Maurer M, Memeo L, Su T, Wang $\mathrm{X}$, et al. PIK3CA mutations correlate with hormone receptors, node metastasis, and ERBB2, and are mutually exclusive with PTEN loss in human breast carcinoma. Cancer Res. 2005 Apr;65(7):2554-9.

44 Loibl S, von Minckwitz G, Schneeweiss A Paepke S, Lehmann A, Rezai M, et al. PIK3CA mutations are associated with lower rates of pathologic complete response to anti-human epidermal growth factor receptor 2 (her2) therapy in primary HER2-overexpressing breast cancer. J Clin Oncol. 2014 Oct;32(29): 3212-20.

45 Guarneri V, Dieci MV, Frassoldati A, Maiorana A, Ficarra G, Bettelli S, et al. Prospective Biomarker Analysis of the Randomized CHER-LOB Study Evaluating the Dual AntiHER2 Treatment With Trastuzumab and Lapatinib Plus Chemotherapy as Neoadjuvant Therapy for HER2-Positive Breast Cancer. Oncologist. 2015 Sep;20(9):1001-10.

46 Loi S, Michiels S, Lambrechts D, Fumagalli D, Claes B, Kellokumpu-Lehtinen PL, et al. Somatic mutation profiling and associations with prognosis and trastuzumab benefit in early breast cancer. J Natl Cancer Inst. 2013 Jul;105(13):960-7.

47 Tekesin K, Akar E, Gunes ME, Bayrak S, Ozturk T, Tural D. PTEN loss is a predictive marker for HER2-positive metastatic breast cancer patients treated with trastuzumabbased therapies. J BUON. 2019;24(5):1920-6.

48 Gori S, Sidoni A, Colozza M, Ferri I, Mameli MG, Fenocchio D, et al. EGFR, pMAPK, pAkt and PTEN status by immunohistochemistry: correlation with clinical outcome in HER2positive metastatic breast cancer patients treated with trastuzumab. Ann Oncol. 2009 Apr;20(4):648-54.

49 Perez EA, Dueck AC, McCullough AE, Chen $\mathrm{B}$, Geiger XJ, Jenkins RB, et al. Impact of PTEN protein expression on benefit from adjuvant trastuzumab in early-stage human epidermal growth factor receptor 2-positive breast cancer in the North Central Cancer Treatment Group N9831 trial. J Clin Oncol. 2013 Jun;31(17):2115-22.

50 Nuciforo PG, Aura C, Holmes E, Prudkin L, Jimenez J, Martinez P, et al. Benefit to neoadjuvant anti-human epidermal growth factor receptor 2 (HER2)-targeted therapies in HER2-positive primary breast cancer is independent of phosphatase and tensin homolog deleted from chromosome 10 (PTEN) status. Ann Oncol. 2015 Jul;26(7):1494-500.

51 Bae SB, Cho HD, Oh MH, Lee JH, Jang SH, Hong SA, et al. Expression of Programmed Death Receptor Ligand 1 with High TumorInfiltrating Lymphocytes Is Associated with Better Prognosis in Breast Cancer. J Breast Cancer. 2016 Sep;19(3):242-51. 
52 Tsang JY, Au WL, Lo KY, Ni YB, Hlaing T, Hu $\mathrm{J}$, et al. PD-L1 expression and tumor infiltrating PD-1+ lymphocytes associated with outcome in HER2+ breast cancer patients. Breast Cancer Res Treat. 2017 Feb;162(1):19-30.

53 Kurozumi S, Inoue K, Matsumoto H, Fujii T, Horiguchi J, Oyama T, et al. Clinicopathological values of PD-L1 expression in HER2-positive breast cancer. Sci Rep. 2019 Nov;9(1) 16662.

54 Loi S, Giobbie-Hurder A, Gombos A, Bachelot T, Hui R, Curigliano G, et al.; International Breast Cancer Study Group and the Breast International Group. Pembrolizumab plus trastuzumab in trastuzumab-resistant, advanced, HER2-positive breast cancer (PANACEA): a single-arm, multicentre, phase $1 \mathrm{~b}-2$ trial. Lancet Oncol. 2019 Mar;20(3):371-82.

55 Hou Y, Nitta H, Wei L, Banks PM, Lustberg $\mathrm{M}$, Wesolowski R, et al. PD-L1 expression and CD8-positive $T$ cells are associated with favorable survival in HER2-positive invasive breast cancer. Breast J. 2018 Nov;24(6):911-9.

56 Kochi M, Iwamoto T, Niikura N, Bianchini G, Masuda S, Mizoo T, et al. Tumour-infiltrating lymphocytes (TILs)-related genomic signature predicts chemotherapy response in breast cancer. Breast Cancer Res Treat. 2018 Jan;167(1):39-47.

57 Loi S, Sirtaine N, Piette F, Salgado R, Viale G, Van Eenoo F, et al. Prognostic and predictive value of tumor-infiltrating lymphocytes in a phase III randomized adjuvant breast cancer trial in node-positive breast cancer comparing the addition of docetaxel to doxorubicin with doxorubicin-based chemotherapy: BIG 02-98. J Clin Oncol. 2013 Mar;31(7):860-7.
58 Loi S, Michiels S, Salgado R, Sirtaine N, Jose $\mathrm{V}$, Fumagalli D, et al. Tumor infiltrating lymphocytes are prognostic in triple negative breast cancer and predictive for trastuzumab benefit in early breast cancer: results from the FinHER trial. Ann Oncol. 2014 Aug;25(8): 1544-50

59 Ingold Heppner B, Untch M, Denkert C, Pfitzner BM, Lederer B, Schmitt W, et al. Tumor-Infiltrating Lymphocytes: A Predictive and Prognostic Biomarker in NeoadjuvantTreated HER2-Positive Breast Cancer. Clin Cancer Res. 2016 Dec;22(23):5747-54.

60 Salgado R, Denkert C, Campbell C, Savas P, Nuciforo P, Aura C, et al. Tumor-infiltrating lymphocytes and associations with pathological complete response and event-free survival in HER2-positive early-stage breast cancer treated with lapatinib and trastuzumab: a secondary analysis of the NeoALTTO trial. JAMA Oncol. 2015 Jul;1(4):448-54.

61 Liu S, Mou E, Zeng S, Wang L, Dong H, Ji J, et al. Therapeutic effect of trastuzumab in neoadjuvant-treated HER2-positive breast cancer with low infiltrating level of tumor-infiltrating lymphocytes. Cancer Manag Res. 2020 May; 12:3145-53.

62 Liu S, Lachapelle J, Leung S, Gao D, Foulkes WD, Nielsen TO. CD8+ lymphocyte infiltration is an independent favorable prognostic indicator in basal-like breast cancer. Breast Cancer Res. 2012 Mar;14(2):R48.

63 Schmid P, Rugo HS, Adams S, Schneeweiss A, Barrios CH, Iwata $\mathrm{H}$, et al.; IMpassion130 Investigators. Atezolizumab plus nab-paclitaxel as first-line treatment for unresectable, locally advanced or metastatic triple-negative breast cancer (IMpassion130): updated efficacy results from a randomised, double-blind, placebo-controlled, phase 3 trial. Lancet Oncol. 2020 Jan;21(1):44-59.
64 Mavaddat N, Barrowdale D, Andrulis IL, Domchek SM, Eccles D, Nevanlinna H, et al. HEBON; EMBRACE; GEMO Study Collaborators; kConFab Investigators; SWE-BRCA Collaborators; Consortium of Investigators of Modifiers of BRCA1/2. Pathology of breast and ovarian cancers among BRCA1 and BRCA2 mutation carriers: results from the Consortium of Investigators of Modifiers of BRCA1/2 (CIMBA). Cancer Epidemiol Biomarkers Prev. 2012 Jan;21(1):134-47.

65 Evans DG, Lalloo F, Howell S, Verhoef S, Woodward ER, Howell A. Low prevalence of HER2 positivity amongst BRCA1 and BRCA2 mutation carriers and in primary BRCA screens. Breast Cancer Res Treat. 2016 Feb; 155(3):597-601.

66 McGuire A, Brown JA, Kerin MJ. Metastatic breast cancer: the potential of miRNA for diagnosis and treatment monitoring. Cancer Metastasis Rev. 2015 Mar;34(1):145-55.

67 Lowery AJ, Miller N, Devaney A, McNeill RE, Davoren PA, Lemetre C, et al. MicroRNA signatures predict oestrogen receptor, progesterone receptor and HER2/neu receptor status in breast cancer. Breast Cancer Res. 2009; 11(3):R27.

68 Miller TE, Ghoshal K, Ramaswamy B, Roy S, Datta J, Shapiro CL, et al. MicroRNA-221/222 confers tamoxifen resistance in breast cancer by targeting p27Kip1. J Biol Chem. 2008 Oct; 283(44):29897-903. 